\title{
Thiopurine-S-Methyltransferase Gene Polymorphism and Drug-related Toxicity in Children Treated for Acute Leukemia and Non-Hodgkin's Lymphoma
}

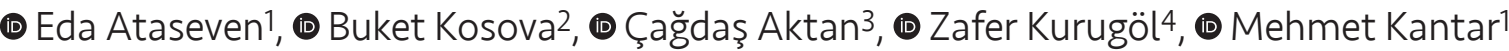 \\ ${ }^{1}$ Ege University Faculty of Medicine, Department of Pediatrics, Division of Pediatric Hematology and Oncology, İzmir, Turkey \\ ${ }^{2}$ Ege University Faculty of Medicine, Department of Medical Biology, Izmir, Turkey \\ ${ }^{3}$ Beykent University Faculty of Medicine, Department of Medical Biology, İstanbul, Turkey \\ ${ }^{4}$ Ege University Faculty of Medicine, Department of Pediatrics, Division of Outpatient Clinic, Izmir, Turkey
}

\begin{abstract}
Aim: Thiopurine S-methyltransferase (TPMT) is an essential enzyme in the metabolism of thiopurine drugs, and its activity may change due to different polymorphisms in the TPMT gene. The TPMT gene has different genetic polymorphisms in different ethnic groups. This study aimed to determine the frequency of TPMT polymorphisms in children with acute leukemia/non-Hodgkin lymphoma (AL/NHL) and healthy children and to evaluate their association with severe toxicities in the study population.

Materials and Methods: Sixty-seven pediatric AL/NHL patients and 84 healthy children were evaluated. Genotyping for the TPMT*2, TPMT*3A TPMT*3B, TPMT*3C, TPMT*4, TPMT*5, TPMT*6, and TPMT*7 alleles were performed by the real-time polymerase chain reaction technique. The number of grade 3 or higher hematologic and hepatic toxicities were recorded from the patient charts.

Results: In the AL/NHL patients, we found that the patients had generally wild-type TPMT*1 allele in $80.6 \%$, whereas TPMT*2 (238G>C) was seen in $1.5 \%$, TPMT $^{*} 3 \mathrm{~A}$ (c.460G $>\mathrm{A}$ and c.719A $>\mathrm{G}$ ) in $0 \%$, and TPMT*3B polymorphisms $(460 \mathrm{G}>\mathrm{A}$ ) in $17.9 \%$. We found wild-type TPMT*1 allele in $98.8 \%$ and TPMT*3B polymorphisms $(460 \mathrm{G}>\mathrm{A})$ in $1.2 \%$ of the healthy volunteers. Grade $\geq 3$ myelosuppression developed in $22 / 54$ patients with the wild type allele, and it developed in 5/12 patients with TPMT*3B allele. Six (8.9\%) patients had grade $\geq 3$ aspartate aminotransferase elevations, 17 (25\%) patients had grade $\geq 3$ alanine transaminase elevations (1-5 times), and 42 patients had (62.6\%) grade $\geq 3$ total bilirubin elevations.

Conclusion: TPMT*3B polymorphism was the most common allele detected in our study group. This allele frequency is very high in comparison to other studies from our country and it was over-represented in comparison to the healthy volunteers. We did not find any relationship between severe hematologic/hepatic toxicities and TPMT gene polymorphisms.
\end{abstract}

Keywords: Thiopurine S-methyltransferase, leukemia, lymphoma, polymorphism, toxicity, childhood 


\section{Introduction}

Thiopurine drugs, such as 6-mercaptopurine (6-MP) and 6-thioguanine (6-TG), are cytotoxic agents used to treat acute leukemia and non-Hodgkin's lymphoma (NHL). Oral 6-MP is a mainstay of the maintenance therapy of acute lymphoblastic leukemia (ALL) and lymphoblastic lymphomas (LLs). Oral 6-TC is another thiopurine drug used in the maintenance treatment of acute myeloid leukemia (AML). One of the most frequent adverse effects of thiopurine drugs is myelosuppression, which is usually reversed by decreasing the dosage of the drug.

Thiopurine S-Methyltransferase (TPMT) is a cytosolic enzyme that converts thiopurine drugs into inactive metabolites via methylation reactions and it provides protection against 6-MP/6-TG drug toxicity (1). Usually, 90\% of patients have normal TPMT activity. However, in $10 \%$ of patients, TPMT enzyme activity may be different due to different TPMT gene polymorphisms. Patients who carry homozygous TPMT gene polymorphisms with two nonfunctional TPMT genes (0.3\%) have no TPMT enzyme activity and may experience life-threatening myelosuppression when taking thiopurine drugs $(1,2)$. Patients with heterozygous mutations with one non-functional allele have intermediate enzyme activity and have a higher risk for toxicity than normal patients, but $40-70 \%$ can tolerate these drugs. Due to these life-threatening toxicities, the Clinical Pharmacogenetics Implementation Consortium (2013) and the US Food and Drug Administration (2015) recommended testing for TPMT gene polymorphism before starting mercaptopurine treatment and adjusting dosages according to gene status (2).

The TPMT gene is located on chromosome 6p22.3, and more than 23 single nucleotide polymorphisms have been reported (3). TPMT 1 (wild-type) allele codes the normal active enzyme. The most common alleles responsible for deficiency are TPMT 2 (c.238G $>$ C), TPMT 3A (c.460G $>$ A and c.719A>C), and TPMT 3C (c.719G>A). These account for $80-95 \%$ of deficient cases. TPMT 3A and 3B polymorphisms may result in an apparent lack of TPMT enzyme activity. On the other hand, TPMT 2 and TPMT 3C polymorphisms do not affect enzyme activity, but they result in a prominent decrease in TPMT protein levels $(4,5)$. Therefore, patients with $A L L, A M L$, and $L L$ may have different sensitivities to thiopurines as a result of these genetic polymorphisms in the TPMT gene.

TPMT has different genetic polymorphisms in different ethnic groups. Therefore, we aimed to determine the frequency and type of TPMT polymorphisms in our study population and assess its relationship with the hematologic and hepatic toxicities of 6-MP and 6-TC.

\section{Materials and Methods}

\section{Subjects}

This study included patients with leukemia, lymphoma, and healthy subjects as a control group. The healthy subjects were collected from those patients who were seen for any reason other than malignancy in the general pediatrics outpatient service. The local ethics committee approved this study, and informed consent was obtained from the parents.

Treatment protocols were ALL-Berlin-FranfurtMünster-90 (BFM), ALL-BFM-95, and Türkiye ALL-2000 for $A L L$ in consecutive years, while AML-BFM-93 was used for AML, and NHL-BFM-90 and 95 were used for LL. In the maintenance treatment of $A L L$ and $L L, 6-M P$ was given at a dosage of $50 \mathrm{mg} / \mathrm{m}^{2} /$ day and oral methotrexate was given weekly (total treatment duration two years for ALL). In the AML maintenance treatment, 6-TC was given at a dosage of $40 \mathrm{mg} / \mathrm{m}^{2} /$ day for one year.

In the maintenance protocols, patients were given $100 \%$ of the estimated drug dosage if white blood cell (WBC) counts were between 2,000-3,000/ $\mathrm{mm}^{3}$ and $50 \%$ of the dosage if WBC counts were between 1,000-2,000/ $\mathrm{mm}^{3}$. The drug is withdrawn in cases where WBC is less than $1,000 / \mathrm{mm}^{3}$. Similarly, the drug is withdrawn in cases where hepatotoxicity is $\geq 3$ grade. The number of grade $\geq 3$ hematologic (Hemoglobin, WBC, platelet counts) and hepatic toxicities [serum alanine transaminase (ALT), aspartate aminotransferase (AST), total bilirubin levels] were obtained from the patient charts by the investigators.

We used the Common Toxicity Criteria version 5 recommended by the National Cancer Institute for hematological and hepatic toxicity. The grades explain the severity of toxicity; Grade 1: Mild, Grade 2: Moderate, Grade 3: Severe, Grade 4: Life-threatening, Grade 5: Death (6).

The patients were followed up at the outpatient service bi-weekly. The highest value measured in each month was considered to be the toxicity value.

\section{DNA Isolation and TPMT Genotyping}

Genomic DNA was extracted from all subjects peripheral blood leukocytes using the high pure polymerase chain reaction (PCR) template preparation kit (Roche Applied Science, Mannheim, Germany) and stored at $-20{ }^{\circ} \mathrm{C}$ until use. Genotyping for the TPMT*2, TPMT*3A, 
TPMT*3B, TPMT*3C, TPMT*4, TPMT*5, TPMT*6 and TPMT*7 alleles was performed according to the modified protocol of Tai et al. (4) by means of real-time PCR using the Light Cycler ${ }^{\circledR}$ v.2.0 instrument (Roche Applied Science, Mannheim, Germany). For this purpose, specific primers and hybridization probes (TIB MOLBIOL, Berlin, Germany) for the analyzed alleles were used combined with the Light Cycler DNA Master Hybridization Probes Kit (Roche Applied Science, Mannheim, Germany). Polymorphic alleles were identified by the specific melting temperature $(\mathrm{Tm})$ of the resulting amplicons.

\section{Statistical Analysis}

Data were analyzed using SPSS software (version 21.0). The means of the groups were analyzed using nonparametric tests. Correlation of the TPMT polymorphisms with different parameters was performed using the chisquare or Fisher's exact tests, and a p-value of $<0.05$ was considered statistically significant.

\section{Results}

The study group consisted of 67 patients ( 43 male, 24 female), and the mean age was 8.1 years (1-18 years). The diagnosis was ALL in 42, AML in 5, and LL in 20 patients. The control group consisted of 84 healthy children (34 male and 50 female) with a mean age of 9.5 years ( $1-18$ years). Patient characteristics are given in Table 1.

\begin{tabular}{|l|l|l|}
\hline \multicolumn{3}{|l|}{ Table I. Characteristic features of the patients } \\
\hline & Patients (n) & Control group (n) \\
\hline Age, years & $8.1(1-18)$ & $9.5(1-18)$ \\
\hline Gender & 43 & 34 \\
\hline Male & 24 & 50 \\
\hline Female & \multicolumn{2}{|l}{} \\
\hline Disease & 42 & - \\
\hline ALL & 5 & - \\
AML & 20 & - \\
NHL &
\end{tabular}

ALL: Acute lymphoblastic leukemia, AML: Acute myeloid leukemia, NHL: NonHodgkin lymphoma
In the genotyping, we found that the patients in the study group had generally wild-type TPMT $\left({ }^{*} 1\right)$ allele at a prevalence of $80.6 \%$, TPMT $^{*} 2(\mathrm{G} 238 \mathrm{C})$ at a prelence of 1.5 $\%$ and TPMT*3B polymorphisms (G460A) at a prevalence of $17.9 \%$ (Table II). The polymorphisms detected were heterozygous mutations, and no homozygous mutations were detected. Other polymorphisms including TPMT*3A, TPMT*3C (A719G), TPMT*3D, TPMT*4 (G-A), TPMT*5 (T146C), TPMT*6 (A539T) and TPMT*7 (T681G) were not detected.

In the control group, wild-type TPMT $\left({ }^{*} 1\right)$ was $98.8 \%$ and TPMT*3B was $1.2 \%$. Other polymorphisms were not detected. The leukemia-lymphoma patients were found to have less wild-type TPMT but more TPMT*3B polymorphism ( $p=0.0001$ and 0.0001) (Table II).

As for hematologic toxicity, only one patient (1.5\%) developed grade $\geq 3$ anemia ( 2 times) during maintenance treatment, and this patient had a wild-type TPMT allele. However, 28 patients (41.8\%) developed grade $\geq 3$ leukopenia. Of these patients, 22 of them had wild-type TPMT allele, 5 of them had TPMT*3B polymorphism, and one of them had TPMT*2 polymorphism. None of the patients developed grade $\geq 3$ thrombocytopenia (Table III).

As for severe hepatic toxicity, six (8.9\%) patients had grade $\geq 3$ AST elevations, while 17 (25\%) patients had grade $\geq 3$ ALT elevations (1-5 times), and 42 patients (62.6\%) had grade $\geq 3$ total bilirubin elevations (1-23 times) (Table III).

We did not find any relationship between the numbers of severe (grade $\geq 3$ ) hematologic/hepatic toxicities and TPMT gene polymorphisms in the statistical analysis.

\section{Discussion}

Thiopurine drugs, such as 6-MP and 6-TC are a mainstay of the maintenance therapy of acute leukemia and LLs. TPMT enzyme catalyzes the methylation and inactivation of thiopurine drugs. The enzyme activity is influenced by polymorphisms in the TPMT gene and TPMT has different genetic polymorphisms in different ethnic groups. It is important to know TPMT polymorphisms in the population

Table II. TPMT polymorphism distribution of the patients

\begin{tabular}{|l|l|l|l|}
\hline TPMT allele & SNP position & $\begin{array}{l}\text { Genotype distrubition } \\
\mathbf{n = 6 7} \text { (patient) }\end{array}$ & $\begin{array}{l}\text { Genotype distrubition } \\
\mathbf{n = 8 6} \text { (control) }\end{array}$ \\
\hline TPMT 1 & Wild & $54(80.6)$ & $85(98.8)$ \\
\hline TPMT 2 & 238 G $>$ C & $1(1.5)$ & 0 \\
\hline TPMT 3B & 460 G > A & $12(17.9)$ & $1(1.2)$ \\
\hline
\end{tabular}

TPMT: Thiopurine S-methyltransferase, SNP: Single-nucleotide polymorphism 
Table III. Grade $\geq 3$ hematologic/hepatic toxicities in patients according to TPMT polymorphism ( $n=67)$

\begin{tabular}{|l|l|l|l|l|l|l|}
\hline & ALT (n) & AST (n) & T. Bil & Anemia (n) & Leukopenia (n) & Trombocytopenia (n) \\
\hline TPMT 1 & 11 & 5 & 31 & 1 & 22 & 0 \\
\hline TPMT 2 & 0 & 0 & 1 & 0 & 1 & 0 \\
\hline TPMT 3B & 6 & 1 & 10 & 0 & 5 & 0 \\
\hline
\end{tabular}

to evaluate the safety of these drugs. Therefore, we aimed to determine the frequency and type of TPMT polymorphisms in Turkish children. The most common polymorphism was $\mathrm{TPMT}^{*} 3 \mathrm{~B}$ allele in healthy Turkish children (1.2\%). Other polymorphisms $\left(\mathrm{TPMT}^{*} 2, \mathrm{TPMT}^{*} 3 \mathrm{~B}, \mathrm{TPMT}^{*} 3 \mathrm{~A}, \mathrm{TPMT}^{*} 3 \mathrm{C}\right.$, TPMT*3D, TPMT*4, TPMT*5, TPMT*6, TPMT*7) were not determined. There is a limited data about the TPMT polymorphisms in healthy Turkish children. Sayitoğlu et al. (7) analyzed TPMT genotypes in healthy Turkish individuals and detected the prevalence of the $\mathrm{TPMT}^{*} 2$ to be $2 \%$, $\mathrm{TPMT}^{*} 3 \mathrm{~B}$ to be $0 \%$, and TPMT*3C to be $1.4 \%$. However, this is different from our results. We did not detect TPMT*2 and $\mathrm{TPMT}^{*} 3 \mathrm{C}$ allels.

In the leukemia-lymphoma group, we found that leukemic children had wild type TPMT allele (TPMT*1) in $80.6 \%$, TPMT $^{*} 3 \mathrm{~B}$ in $17.9 \%$, and $\mathrm{TPMT}^{*} 2$ in $1.5 \%$. Unlike other studies, $\mathrm{TPMT}^{*} 3 \mathrm{~B}$ allele frequency was very high in our patients. TPMT*3B is a rare allele that is usually absent in many populations. Tumer et al. (8) studied 106 Turkish children with ALL and detected TPMT*2 in $0 \%$, TPMT*3B in $^{*}$ $0.9 \%$, and TPMT*3C in 0.9\%. Similarly, Akın et al. (9) studied 169 children with leukemia and detected TPMT*2 in 0\%, TPMT*3A in 1.7\%, TPMT*3B in 1.7\%, and TPMT*3C in $2.4 \%$. We did not detect TPMT*3C and TPMT*3A in our population. This difference may be related to our study population. Our population was recruited from Western Anatolia. However, other studies were recruited from the Marmara region of Central Anatolia. Only a few studies have reported TPMT*3B polymorphism as high in the literature. Moreno-Guerrero et al. (10) studied Mexican children with different cancer types and reported $\mathrm{TPMT}^{*} 3 \mathrm{~B}$ polymorphism at a rate of $7.5 \%$. However, another study from Mexico reported TPMT*3B to be $0.1 \%$ in leukemia patients (11). According to these results, we thought there might be regional differences within the same country. In Europe, TPMT-3B was only reported in the Spanish population to be $1.5 \%$, and other studies from our country at a prevalence of 0.9-1.7\% (7-9,12). Among Asian countries, an Iranian study reported TPMT*3B allele to be $1.6 \%$ in the healthy population (13).

The increased TPMT*3B allele frequency in the patient group compared to healthy children is striking in our study group. This result raises the question of whether these polymorphisms might affect leukemia susceptibility. We know that folate metabolism plays an important role in DNA synthesis and methylation. Deviations in the folate metabolism resulting from polymorphisms in genes encoding folate-dependent enzymes may affect susceptibility to leukemia. One of the most extensive series from Mexico studied 849 patients (428 ALL, 421 non-ALL), and they found TPMT polymorphism frequency to be higher in ALL patients but did not find it statistically significant (11). Previous studies from the Mexican population also reported TPMT polymorphisms are not a risk factor for ALL $(14,15)$.

In our study, TPMT*3C allele was not detected. However, $\mathrm{TPMT}^{*} 3 \mathrm{C}$ is the most frequent allele in Asian and African populations (16). Leukemic children from Singapore were reported to have $\mathrm{TPMT}^{*} 3 \mathrm{C}$ allele at a rate of $3 \%$ in the Chinese population, $2.3 \%$ in Malaysians, whereas children from Thailand have TPMT*3C at a rate of $11 \%(17,18)$.

TPMT*$^{*} \mathrm{~A}$ is the most common allele in the Caucasian and American populations but was not detected in our study. Lennard et al. (19) evaluated 1,320 children with ALL in England and detected TPMT*3A at a rate of 4.5\%, and $\mathrm{TPMT}^{*} \mathrm{BB}$ at a rate of $0 \%$. For the Serbian population, $\mathrm{TPMT}^{*} 3 \mathrm{~A}$ allele frequency was reported as $3.2 \%$ while TPMT $^{*} 2$ was $0.2 \%$, and TPMT*3B was $0.5 \%$ (20).

TPMT $^{*} 2$ is another less common allele. This allele has been detected $0-0.1 \%$ in Asian populations and $0.1-0.85 \%$ in European populations $(11,13)$. On the other hand, two larger sample-sized studies in Iran reported this allele at a rate of 2.16-3.93\% (13,21). In our study, TPMT*2 frequency was 1.5\%.

As for drug toxicity, theoretically, it can be assumed that children with heterozygous or homozygous polymorphisms might experience more hematologic or hepatic toxicities of 6-MP and 6-TG as a result of decreased enzyme activity, decreased thiopurine clearance from plasma, and accumulation of the drug in the body. There are many studies regarding thiopurine dosage and its relevance with TPMT alleles, but this research is very limited in the pediatric age group. 
Relling et al. (22) showed that 6-MP dose reductions due to toxicity, rates of thrombocytopenia, neutropenia, and hospitalization due to infection were highest among those patients homozygous for mutant TPMT, intermediate among heterozygous mutations, and lowest among wildtype patients. Interestingly, they showed hepatotoxicity tends to be more frequent in wild-type patients. We speculate that methylated metabolites probably contribute to hepatotoxicity. El-Rashedy et al. (23) had similar results when they reported TPMT mutant patients to have a high risk of hematological toxicity, but there was no statistically significant increase in hepatotoxicity. However, we did not find any relationship between the hematologic/hepatic toxicity and TPMT gene polymorphisms. Similar to our findings, Ayesh et al. (24) evaluated 56 ALL patients in the Palestinian territories. Out of 14 myelosuppression cases, only one patient had TPMT polymorphism and this reported myelosuppression could not be explained only by the existence of polymorphism. Farfan et al. (25) studied 103 Chilean ALL children and detected polymorphisms of TPMT $^{*} 3 \mathrm{~A}$ in $7 \%$, and TPMT*3C in $1 \%$. However, they did not find any relationship between toxicity and wild-type or mutant alleles (25). These results suggest the effect of other genetic factors or other polymorphisms in the development of toxicity.

In the last decade, several studies have shown that there are many genes involved in thiopurine metabolism and drug intolerance, such as BCC4, ABCC5, IMPDH1, ITPA, SLC28A3, XDH and NUDT15 (26). Especially, NUDT15 and ITPA are the most important of these. NUDT15 is an enzyme involved in the metabolism of thiopurine drugs and has shown a strong association with thiopurine intolerance. Yang et al. (27) reported that NUDT15 polymorphism causes susceptibility to thiopurine-induced myelosuppression. However, Moradveisi et al. (28) reported that TPMT and NUDT15 polymorphisms are quite rare and, therefore, the ITPA polymorphism is important. They found that the ITPA polymorphism was more common in their study group and that there was a significant relationship between the ITPA polymorphism and the dosing intensity of 6-MP (28). Interestingly, a study from our country showed that TPMT and ITPA variants were rare in our population, but SLC01B1 polymorphisms were common, and these variants were associated with thiopurine intolerance (29).

\section{Conclusion}

In conclusion, TPMT gene polymorphisms are important because of life-threatening complications during 6-MP and 6-TC therapies. TPMT*3B polymorphism, which is described as being rare in the published data, was the most common allele detected in our $\mathrm{AL} / \mathrm{NHL}$ patient group. Our study showed different results from previous studies in Turkey. We did not find a possible relationship between polymorphisms and severe hematologic or hepatic toxicities.

\section{Ethics}

Ethics Committee Approval: The Local Ethics Committee of Ege University Faculty of Medicine approved this study (approval number: 05-4/5, date: 28.04.2021).

Informed Consent: Informed consent was obtained from the parents.

Peer-review: Externally peer-reviewed.

\section{Authorship Contributions}

Concept and Design: E.A., B.K., C..A., Z.K., M.K., Data Collection or Processing: B.K., Ç.A., Analysis Interpretation: E.A., Literature Search: M.K., Writing: E.A., M.K.

Conflict of Interest: The authors declare that they have no conflict of interest.

Financial Disclosure: The authors declared that this study received no financial support.

\section{References}

1. Azimi F, Jafariyan M, Khatami S, Mortazavi Y, Azad M. Assessment of thiopurine-based drugs according to thiopurineS-methyltransferase genotype in patients with acute lymphoblastic leukemia. Iran J Ped Hematol Oncol 2014; 4:32-8.

2. Dean L, Kane M. Mercaptopurine Therapy and TPMT and NUDT15 Genotype. In: Pratt VM, Scott SA, Pirmohamed M, et al (eds). Medical Genetics Summaries: Bethesda, National Center for Biotechnology Information (US), 2012.

3. Szumlanski C, Otterness D, Her C, et al. Thiopurine methyltransferase pharmacogenetics: human gene cloning and characterization of a common polymorphism. DNA Cell Biol 1996; 15:17-30.

4. Tai HL, Krynetski EY, Schuetz EG, Yanishevski Y, Evans WE. Enhanced proteolysis of thiopurine S-methyltransferase (TPMT) en-coded by mutant alleles in humans (TPMT*3A, TPMT*2): mechanisms for the genetic polymorphism of TPMT activity. Proc Natl Acad Sci USA 1997; 10:94:6444-9.

5. Wang L, Nguyen TV, McLaughlin RW, Sikkink LA, RamirezAlvarado M, Weinshilboum RM. Human thiopurine S-methyltransferase pharmacogenetics: variant allozyme misfolding and aggresome formation. Proc Natl Acad Sci U S A 2005; 28:102:9394-9.

6. https://ctep.cancer.gov/protocoldevelopment/electronic applications/docs/ctcae v5 quick reference 5 $\times 7 . p d f$ Published: November 27, 2017

7. Sayitoğlu MA, Yıldız I, Hatırnaz Ö, Özbek U. Common Cytochrome p4503A (CYP3A4 and CYP3A5) and Thiopurine S-methyl trans-ferase (TPMT) polymorphisms in Turkish population. Turk J Med Sci 2006; 36:11-5. 
8. Tumer TB, Ulusoy G, Adali O, Sahin G, Gozdasoglu S, Arinç E. The low frequency of defective TPMT alleles in Turkish population: a study on pediatric patients with acute lymphoblastic leukemia. Am J Hematol 2007; 82:906-10.

9. Akın DF, Aşlar-Öner D, Kürekçi E, Akar N. Frequency of thiopurine S-methyltransferase gene variations in Turkish children with acute leukemia. Turk J Pediatr 2018; 60:147-52.

10. Moreno-Guerrero SS, Ramírez-Pacheco A, Dorantes-Acosta EM, Medina-Sanson A. Analysis of genetic polymorphisms of thiopurine S-methyltransferase (TPMT) in Mexican pediatric patients with cancer. Rev Invest Clin 2013; 65:156-64.

11. Jiménez-Morales S, Ramírez-Florencio M, Mejía-Aranguré JM, et al. Analysis of thiopurine S-methyltransferase deficient alleles in acute lymphoblastic leukemia patients in Mexican patients. Arch Med Res 2016; 47:615-22.

12. Corominas $H$, Domènech $M$, del Río $E$, Gich $I$, Domingo $P$, Baiget M. Frequency of thiopurine S-methyltransferase alleles in different ethnic groups living in Spain. Med Clin (Barc) 2006; 126:410-2.

13. Bahari A, Hashemi M, Bari Z, Moazeni-Roodi A, Kaykhaei MA, Narouie B. Frequency of thiopurine S-methyltransferase (TPMT) alleles in southeast Iranian population. Nucleosides Nucleotides Nucleic Acids 2010; 29:237-44.

14. Gutiérrez-Álvarez $O$, Lares-Asseff I, Galaviz-Hernández $C$, et al. Involvement of MTHFR and TPMT genes in susceptibility to child-hood acute lymphoblastic leukemia (ALL) in Mexicans. Drug Metab Pers Ther 2016; 31:41-6.

15. Taja-Chayeb L, Vidal-Millán $S$, Gutiérrez $O$, OstroskyWegman P, Dueñas-González A, Candelaria M. Thiopurine S-methyltransferase gene (TMPT) polymorphisms in a Mexican population of healthy individuals and leukemic patients. Med Oncol 2008; 25:56-62

16. Hamdy SI, Hiratsuka M, Narahara K, et al. Genotype and allele frequencies of TPMT, NAT2, GST, SULT1A1 and MDR-1 in the Egyptian population. Br J Clin Pharmacol 2003; 55:560-9.

17. Kham SK, Tan PL, Tay AH, Heng CK, Yeoh AE, Quah TC. Thiopurine methyltransferase polymorphisms in a multiracial asian population and children with acute lymphoblastic leukemia. J Pediatr Hematol Oncol 2002; 24:353-9.

18. Hongeng S, Sasanakul W, Chuansumrit A, Pakakasama S, Chattananon A, Hathirat P. Frequency of thiopurine S-methyltransferase genetic variation in Thai children with acute leukemia. Med Pediatr Oncol 2000; 35:410-4.

19. Lennard L, Cartwright CS, Wade R, Richards SM, Vora A. Thiopurine methyltransferase genotype-phenotype discordance and thiopu-rine active metabolite formation in childhood acute lymphoblastic leukemia. Br J Clin Pharmacol 2013; 76:125-36.

20. Dokmanovic L, Urosevic J, Janic $D$, et al. Analysis of thiopurine $\mathrm{S}$-methyltransferase polymorphism in the population of Serbia and Montenegro and mercaptopurine therapy tolerance in childhood acute lymphoblastic leukemia. Ther Drug Monit 2006; 28:800-6.

21. Azad M, Kaviani S, Soleimani M, Noruzinia M, Hajfathali A. Common Polymorphism's Analysis of Thiopurine S-Methyltransferase (TPMT) in Iranian Population. Yakhteh Medical Journal 2009; 11:311-6.

22. Relling MV, Hancock ML, Rivera GK, et al. Mercaptopurine therapy intolerance and heterozygosity at the thiopurine S-methyltransferase gene locus. I Natl Cancer Inst 1999; 91:2001-8

23. El-Rashedy FH, Ragab SM, Dawood AA, Temraz SA. Clinical implication of thiopurine methyltransferase polymorphism in children with acute lymphoblastic leukemia: a preliminary Egyptian study. Indian I Med Paediatr Oncol 2015; 36:265-70.

24. Ayesh BM, Harb WM, Abed AA. Thiopurine methyltransferase genotyping in Palestinian childhood acute lymphoblastic leukemia patients. BMC Hematol 2013; 13:3.

25. Farfan MJ, Salas C, Canales C, et al. Prevalence of TPMT and ITPA gene polymorphisms and effect on mercaptopurine dosage in Chilean children with acute lymphoblastic leukemia. BMC Cancer 2014; 14:299.

26. Zgheib NK, Akika R, Mahfouz R, et al. NUDT15 and TPMT genetic polymorphisms are related to 6-mercaptopurine intolerance in children treated for acute lymphoblastic leukemia at the Children's Cancer Center of Lebanon. Pediatr Blood Cancer 2017; 64:146-50.

27. Yang J, Landier W, Yang W, et al. Inherited NUDT15 variant is a genetic determinant of mercaptopurine intolerance in children with acute lymphoblastic leukemia. I Clin Oncol 2015; 33:123542.

28. Moradveisi B, Muwakkit S, Zamani F, Ghaderi E, Mohammadi E, Zgheib NK. ITPA, TPMT, and NUDT15 Genetic Polymorphisms Predict 6-Mercaptopurine Toxicity in Middle Eastern Children With Acute Lymphoblastic Leukemia. Front Pharmacol 2019; 10:916.

29. Eldem I, Yavuz D, Cumaoğullari Ö, et al. SLCO1B1 Polymorphisms are Associated With Drug Intolerance in Childhood Leukemia Maintenance Therapy. J Pediatr Hematol Oncol 2018; 40:289-94. 